\title{
Lidil
}

Revue de linguistique et de didactique des langues

43 | 2011

Le rapport au savoir dans les discours professionnels

\section{L'utilisation du tableau noir : effet des routines sur la construction d'un nouveau savoir didactique}

Can an educational gesture hide another one? The use of the blackboard: effects of teaching routines on new didactic knowledge construction

\section{Solveig Lepoire-Duc}

\section{(2) OpenEdition}

\section{Journals}

Édition électronique

URL : http://journals.openedition.org/lidil/3108

DOI : 10.4000/lidil.3108

ISSN : $1960-6052$

Éditeur

UGA Éditions/Université Grenoble Alpes

Édition imprimée

Date de publication : 30 mai 2011

Pagination : $57-73$

ISBN : 978-2-84310-201-1

ISSN : $1146-6480$

\section{Référence électronique}

Solveig Lepoire-Duc, « L'utilisation du tableau noir : effet des routines sur la construction d'un nouveau savoir didactique », Lidil [En ligne], 43 | 2011, mis en ligne le 30 novembre 2012, consulté le 30 avril 2019. URL : http://journals.openedition.org/lidil/3108 ; DOI : 10.4000/lidil.3108 


\title{
L'utilisation du tableau noir : effet des routines sur la construction d'un nouveau savoir didactique
}

\author{
Solveig Lepoire-Duc*
}

\begin{abstract}
RÉSUMÉ
Incorporer un nouveau geste professionnel à la pratique enseignante peut conduire à reconfigurer des routines, comme l'utilisation du tableau noir. Nous observons l'usage que deux enseignants font de ce dernier pour appliquer, en grammaire, une démarche de conceptualisation qu'ils viennent de découvrir. La comparaison montre que les modalités d'utilisation du tableau ont une incidence sur la mise en œuvre de cette démarche. Elles peuvent modifier la nature de l'activité pour, par exemple, transformer une situation problème en une activité du type «exercice». À l'inverse, le tableau peut stimuler l'activité langagière et cognitive de la classe lorsqu'il est géré comme un brouillon collectif. Ainsi, nous distinguons les «routines pédagogiques » d'utilisation du tableau, transférables à différentes situations d'apprentissage, et les «routines didactiques », spécifiques à un type de démarche.
\end{abstract}

\section{ABSTRACT}

Incorporating a new pedagogical act into teaching may lead to the reconfiguration of routines such as the use of the blackboard. This study observes the way the blackboard is used to apply a "conceptualisation" approach to grammar that the teachers have just discovered. The comparison shows that the types of use to which the blackboard is put have an effect on the application of this approach. They can modify the nature of the activity in order, for example, to transform a problem situation into an "exercise" type activity. Conversely, the blackboard can stimulate language and cognitive activity in the class when it is used as a collective rough draft. A distinction can thus be made between "pedagogical routines" of blackboard use, transferable to different learning situations, and "didactique routines" specific to a type of approach.

* Université Claude Bernard-Lyon 1, IUFM

ICAR UMR 5191, Université Lumière-Lyon 2. 


\section{Incidence de la formation continue sur la reconfiguration des gestes professionnels}

Notre réflexion porte sur la construction de savoirs professionnels d'enseignants du premier degré dans le cadre de la formation continue. Par nature composites, les savoirs professionnels s'appuient sur une série de savoirs scientifiques (disciplinaires et didactiques) et expérientiels, ces derniers étant issus de l'action et non toujours mis en mots (Altet, 2008). L'enjeu de la formation veut que des savoirs nouveaux proposés «de l'extérieur» acquièrent le statut de ressources devant être progressivement incorporées à l'action enseignante (Mayen, 2008). Toutefois, des recherches récentes montrent que ni leur rationalité scientifique ni même leur caractère réglementaire n'accordent d'office à ces savoirs «un droit d'entrée dans l'organisation de l'action» (Mayen, 2008). Au contact avec la réalité du métier, les enseignants doivent les «convertir» et les «reconfigurer» et c'est finalement, conclut Altet (2008, p. 94), «dans l'expérience du travail enseignant [que] se réalise le métissage de ces différents savoirs ». S. Vanhulle (2009, p. 261) insiste sur le rôle des stages qui, en formation initiale, permettent de penser les liens entre la formation scientifique et l'apprentissage par l'expérience, à condition que les experts des différents domaines scientifiques et de la profession collaborent «pour que la pluralité des regards se mette au service de cette intégration sur la base des situations réelles de travail». Les choses se passent différemment en formation continue où l'enseignantstagiaire ne bénéficie d'aucun accompagnement pour reconfigurer ses «formats de travail scolaire» (Bautier, 2006) à la sortie d'un stage. Ceci peut expliquer - en partie au moins - que les connaissances apportées en formation continue se trouvent si difficilement intégrées aux formes d'actions déjà en place. D'où notre problématique qui rejoint celles d'Altet (2008), lorsqu'elle cherche à comprendre les processus de configuration des savoirs dans l'action, et de Mayen (2008, p. 46) lorsqu'il se demande «comment insérer du savoir dans le faire». Notre recherche, qui se situe au carrefour de la didactique professionnelle et de la didactique des disciplines, interroge les effets d'un savoir pratique routinisé - l'utilisation du tableau noir - sur l'intégration dans l'agir enseignant d'un savoir didactique nouveau : une démarche de conceptualisation en grammaire. Cette démarche, reprise à Barth (1987) pour accompagner la résolution de problèmes de langue (Sautot et LepoireDuc, 2010) a été expliquée au cours d'un stage à un groupe de professeurs des écoles volontaires, qui ont accepté d'être filmés en train de la mettre en œuvre. L'observation directe a mis en évidence des 
manières différentes de s'approprier ce nouveau geste professionnel. Nous faisons l'hypothèse qu'un des obstacles qu'ont pu rencontrer ces enseignants pour utiliser la démarche que nous leur proposions tient à sa confrontation avec des gestes professionnels routinisés, notamment l'utilisation du tableau noir.

\section{Deux gestes professionnels corrélés : démarche de conceptualisation de Barth et usage du tableau noir}

\section{La démarche de conceptualisation}

Le modèle proposé par Barth correspond à une démarche intellectuelle qui permet de faire élaborer aux élèves un concept : on leur demande de catégoriser les données d'un corpus pour qu'ils identifient la combinaison d'attributs qui le définissent. Cette démarche d'apprentissage repose sur la comparaison d'exemples «oui» et d'exemples «non» qui se distinguent par la présence ou l'absence d'attributs essentiels déterminant le concept. Cette comparaison, réalisée collectivement, suscite des conflits socio-cognitifs qui se traduisent par des échanges argumentatifs et explicatifs. L'outil proposé par Barth correspond donc à un schème d'actions qui modélise une démarche cognitive et il développe une démarche opératoire susceptible d'apporter des réponses aux dérives du constructivisme qui, selon Bonnette (2006), manque de gestes professionnels «codifiés». Il présente alors un intérêt pour toutes les disciplines scolaires, dont la grammaire.

La démarche de conceptualisation de Barth repose sur l'utilisation d'un langage oralo-graphique ${ }^{1}$, dont les caractéristiques sont relativement normées. Le tableau noir (désormais TN) joue en particulier un rôle central dans cette démarche.

\section{L'utilisation du tableau noir : une pratique professionnelle}

Plusieurs recherches menées sur le TN (Nonnon, 1991, 2000; Hassan, 2008) décrivent un certain nombre de routines d'utilisation récurrentes chez les enseignants. Elles le font apparaitre comme un médiateur d'action qui joue un rôle dans la chronogenèse et la topogenèse des savoirs.

1. Bouchard (1998) met en avant la dimension «oralo-graphique» des interactions en classe où l'oral est en général précédé, suivi ou ponctué par des écrits. 
Le tableau est un outil qui s'inscrit dans des schèmes d'actions partagés entre enseignant et élèves. Mais ce qui peut être considéré comme un outil «est moins le tableau comme objet matériel que l'ensemble des modes de discours et des tâches intellectuelles installés dans la culture d'une microsociété scolaire autour de l'écriture au tableau» (Nonnon, 2000 , p. 95). On en déduit que les modalités d'utilisation du TN sont susceptibles de changer selon les types de tâches intellectuelles et selon les codes de fonctionnement de la classe. Ainsi le TN joue-t-il un rôle dans la structuration des discours et des démarches d'apprentissage, de même qu'à l'inverse certains dispositifs d'apprentissage déterminent des modalités particulières d'utilisation du tableau. L'incorporation de nouvelles démarches d'apprentissage dans l'activité enseignante peut donc susciter la construction de nouveaux gestes professionnels liés à l'utilisation du TN. Mais il s'agit de «savoirs de la pratique» (Altet, 2008), qui correspondent à des savoirs d'expériences. Initialement issus du contact avec d'autres enseignants, ils continuent à se construire de manière implicite dans une logique de la mise en œuvre. Le TN est donc lié à l'action enseignante selon des pratiques typifiées et/ou émergentes (Filliettaz, 2002) et l'on peut supposer que l'introduction d'un nouvel outil d'apprentissage peut faire survenir de nouvelles pratiques, susceptibles d'entrer en conflit avec les routines déjà existantes.

\section{Le tableau noir dans la démarche de conceptualisation}

La démarche de conceptualisation repose sur trois modes de représentation de la connaissance que Barth (1987, p. 95) reprend à Bruner :

- mode enactif (sensori-moteur), celui de l'observation-exploration;

- mode iconique (visuel), qui fait accéder à la clarification;

- mode symbolique, qui permet d'atteindre le stade de l'abstraction.

Les deux premiers relèvent du domaine de l'intuition. En grammaire, ils supposent la possibilité d'observer et de manipuler des énoncés. C'est une première fonction du TN qui est ici sollicitée : il «donne à voir» et sert de support au corpus, au matériau à analyser. L'espace du $\mathrm{TN}$ est dédié à la présentation du corpus écrit et à sa catégorisation en exemples «oui»/exemples «non». Cette entrée binaire peut être remplacée par des variantes proposant des formes de tris plus complexes, présentés, par exemple, dans des tableaux à plus de deux colonnes ${ }^{2}$. La

2. Barth consacre un chapitre à ce type de situation, empruntée à Taba (1962). 
phase d'observation et d'exploration suppose aussi différents types de manipulations : des déplacements, des ajouts, des remplacements qui sont rendus possibles par le caractère effaçable du TN.

Ce premier type d'activité débouche sur la formation de concepts que Barth distingue de l'acquisition de concepts où «il ne s'agit plus de chercher un mode de groupement selon des critères subjectifs mais d'identifier la combinaison de critères selon laquelle un concept est déjà défini » (p.47). Cette procédure, ajoute-t-elle, «nécessite une interaction verbale» destinée à «vérifier la règle de classification». Le mode symbolique induit alors une approche oralo-graphique qui contraint les règles d'utilisation du $\mathrm{TN}$. Ces règles conduisent à créer un second espace, dédié à des prises de notes et aux traces de l'activité métacognitive du groupe, produites lors de l'observation des écrits du premier espace.

\section{Hypothèses de recherche}

Deux enseignants, $\mathrm{N}$ et $\mathrm{V}$, ont choisi d'utiliser la démarche de Barth lors de l'étape de structuration des connaissances dans un apprentissage de la grammaire par résolution de problèmes ${ }^{3}$. Au cours de cette étape, les élèves ont exploré les concepts de «groupe nominal» (classe N) et de «complément essentiel» (classe V) en s'appuyant sur des activités de catégorisation et de mise en tableaux de corpus d'énoncés. Dans la classe $\mathrm{N}$, un tableau de quatre colonnes a été construit pour comprendre «comment apporter des précisions sur un objet que l'on décrit» : noms propres, nom + adjectif, nom + complément du nom, nom + proposition relative. La classe $\mathrm{V}$ a catégorisé des $\mathrm{COD}$ et des $\mathrm{COI}$ dans un tableau à deux colonnes pour repérer la présence de la préposition. Les séquences mises en œuvre ont été filmées et transcrites. Nous avons déjà analysé la classe $\mathrm{N}$ sous l'angle de la didactique de la grammaire (Lepoire et Sautot, 2008, 2009), et mis en évidence qu'une bonne connaissance de la substance grammaticale est une condition sans doute nécessaire, mais non suffisante, pour que les enseignants instaurent des pratiques réflexives d'étude de la langue. La maitrise de gestes professionnels

3. Dans le cadre de la recherche-formation en didactique de la grammaire (DIDAGRAM) menée à l'IUFM de Lyon, nous avons filmé des séquences d'enseignement dans quatre classes multi-âge de cycle 3 dont les enseignants ont choisi d'accompagner leurs élèves dans des démarches de réflexion sur la langue. 
transversaux est également indispensable pour mettre en place de telles pratiques. Un de ces gestes professionnels porte sur la gestion du TN.

Nous faisons donc l'hypothèse que les gestes professionnels d'utilisation du TN ont une incidence sur la mise en œuvre de la démarche de conceptualisation de Barth. L'analyse des séquences filmées sera conduite en vue de répondre aux deux questions suivantes :

1. Peut-on observer une corrélation entre les gestes professionnels d'utilisation du TN et la mise en ouvre de la démarche de Barth?

2. Le recours à cette démarche d'apprentissage est-il compatible avec des « routines» d'utilisation du TN?

\section{Analyse de quelques gestes professionnels d'utilisation du tableau noir}

Hassan (2008) montre que «les écrits du tableau sont à la fois préconstruits par l'enseignant et co-construits avec les élèves» (p. 5). Nous aborderons successivement ces deux modalités d'utilisation du TN.

\section{Le tableau noir pour construire le cadre de l'activité}

Les enseignants anticipent en général la notation au TN des contenus qui bornent la séance : définition de l'activité et institutionnalisation des savoirs. Les deux se retrouvent effectivement dans les classes $\mathrm{N}$ et $\mathrm{V}$, mais traités différemment par les enseignants.

\section{Inscription de l'activité dans l'ergonomie générale du tableau et de la séquence}

Dans les deux classes, le TN sert d'abord de support à l'élaboration progressive de la consigne. Dans la démarche d'apprentissage de Barth (p. 67), celle-ci «a pour but de structurer la tâche et de motiver l'élève en montrant qu'il y a un problème à résoudre ». Dans un premier temps, l'enseignant met explicitement en avant l'existence d'un concept que les élèves vont devoir découvrir. Puis il indique la procédure qui va être utilisée pour comparer les exemples «oui» et «non», avant d'inscrire les premiers au tableau.

Dans les deux classes, la finalité de l'activité et la procédure sont mêlées dans une unique présentation qui sert en même temps d'ancrage dans la séquence. $\mathrm{N}$ et $\mathrm{V}$ débutent la séance par un rappel de ce qui a été fait lors de la séance précédente et annoncent l'objet de celle du jour. 
Cette phase initiale, presque exclusivement orale chez $\mathrm{N}$, fait référence à une fiche de travail distribuée à chaque élève en fin de séance précédente : «il fallait classer ces groupes de mots / on avait eu différents classements / aujourd'hui on va s'intéresser à celui de la grammaire / je vous avais distribué cette fiche-là / je vous réécris un petit peu ce qui est dessus.» Le TN reproduit sommairement les quatre colonnes du tableau de classement des groupes nominaux ${ }^{4}$, les trois premières faisant figurer les premiers exemples «oui»; la dernière est mise en attente par un point d'interrogation :

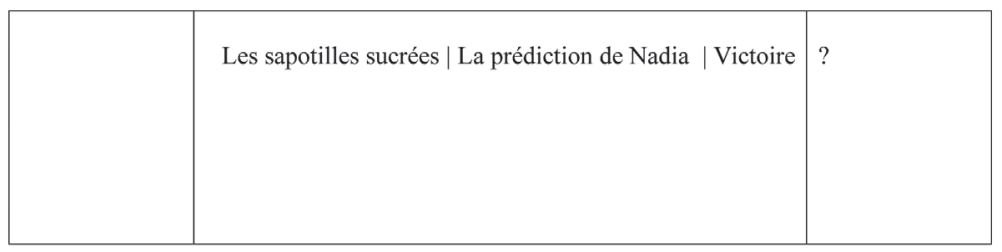

Figure 1. - TN-N1.

Dans la classe de V, les propos de l'enseignant s'accompagnent d'une utilisation importante du TN qui sert de support à un enchainement d'actions pour situer la séance dans l'ensemble de la séquence (TN gauche), noter la consigne par écrit (TN central), reproduire le tableau de classement qui figure sur la fiche distribuée aux élèves (TN droit), recopier la liste des exemples qu'il va falloir classer dans ce tableau (TN central). V met ainsi en œuvre un schème d'action décrit comme typique par Hassan $(2008 \text {, p. } 8)^{5}$ : «l'espace du tableau est divisé en deux ou trois dimensions symboliques [...], chaque espace ayant une fonction dans la progression de la séance.»

4. Les GN sont définis comme apportant des précisions sur le nom. D’où ce classement qui intègre les noms propres.

5. Hassan réfère cette typicité aux enseignants du secondaire, mais elle semble déjà assez prégnante au cycle 3 . 


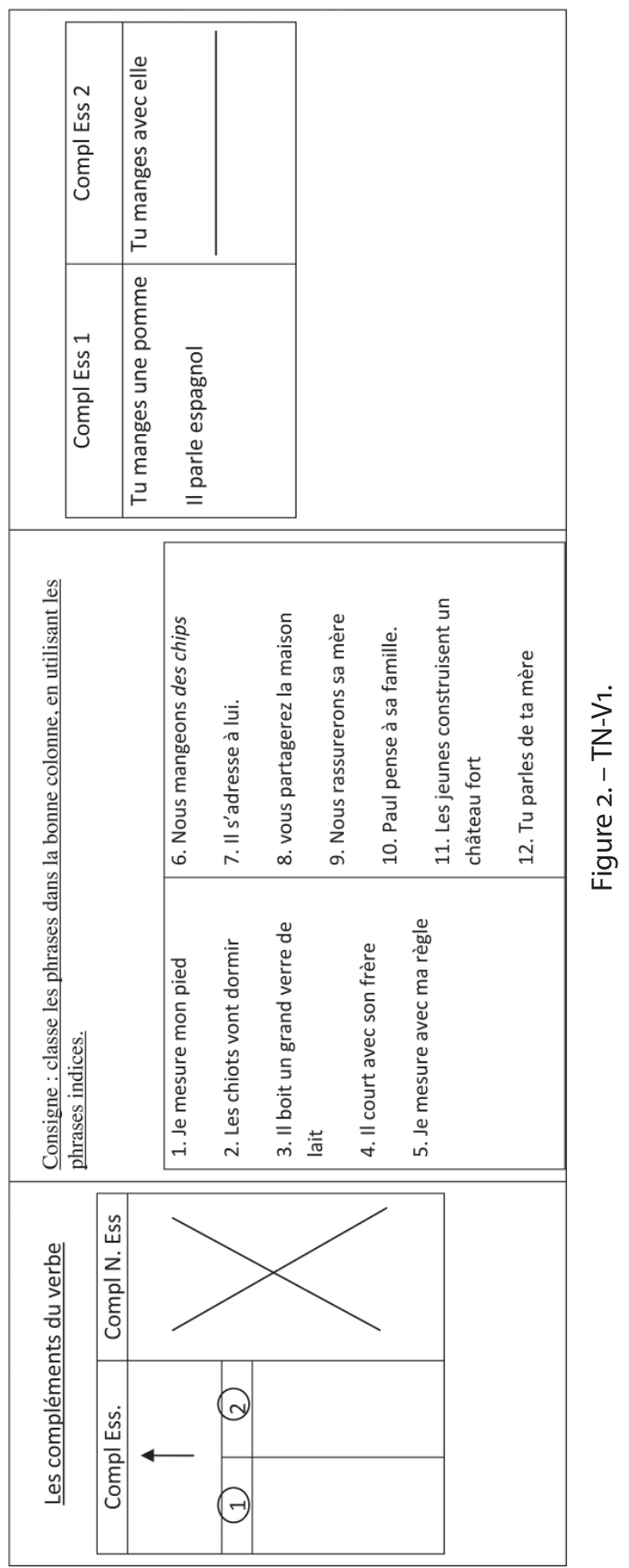


Dans chaque classe, la consigne orale indique un espace problème et une explication à construire (V : «Il va falloir comprendre comment on fait pour refaire une sous-famille dans cette famille-là»; $\mathrm{N}$ : «aujourd'hui on va s'intéresser au classement de la grammaire»), mais aucun des deux enseignants n'a indiqué que le classement a pour finalité de découvrir un nouveau concept. Quant au TN, N l'utilise pour initier l'activité de tri, alors qu'il sert chez $\mathrm{V}$ de support à une «mise en espace» et une «mise en scène pédagogique» de l'activité à venir. Ainsi, $\mathrm{N}$ entre directement dans la démarche de conceptualisation alors que $\mathrm{V}$ recourt à une utilisation du TN qui semble faire partie des habitus de la classe et qui intègre l'activité cible dans une routine du type «exercice de recherche». Cette posture est renforcée par la consigne «classe les phrases dans la bonne colonne», l'adjectif «bonne» prenant une valeur normative de vérité qui n'est pas d'emblée compatible avec la dialectique des exemples «oui»/ «non» censée stimuler la réflexion des élèves.

\section{Le tableau noir comme relais de la consigne orale}

Dans les deux classes, la forme écrite des tableaux complète, de manière implicite, les consignes invitant à effectuer un classement. V a tracé des colonnes devant servir à trier les compléments essentiels en COD et COI. Ce tableau existe en fait en deux exemplaires (cf. fig. 2). Une première fois sur le $\mathrm{TN}$ gauche, sous les en-têtes 1 et 2 ; une seconde fois sur le TN droit, où chaque colonne comporte un ou deux énoncés «pré-classés» de type exemples oui/non, sous les en-têtes Compl Ess 1 et Compl Ess 2. Alors que le premier tableau inscrit l'activité dans l'ergonomie générale de la séquence, le second va servir de support à l'activité elle-même. Cependant, ces en-têtes contiennent des inscriptions qui, bien qu'ayant la même fonction et le même sens, sont réalisées avec des codes différents. Rien n'indique par ailleurs qu'il s'agit d'inscriptions provisoires et que l'activité a pour but de remplacer les signes 1 et 2 par des concepts énoncés verbalement. Le tableau tracé par $\mathrm{N}$ est beaucoup plus sommaire : il figure quatre colonnes comportant chacune un exemple «oui»; les en-têtes sont laissés vides. Ainsi, dans les deux classes, les tableaux sont pré-tracés pour guider l'entrée dans la démarche de conceptualisation. Les premiers exemples «oui»vont permettre d'engager l'activité de catégorisation et les en-têtes serviront à noter les concepts intermédiaires proposés par les élèves. La gestion de ces en-têtes fait toutefois apparaitre une différence notable : $\mathrm{N}$ fait figurer des espaces à remplir sur lesquels va se construire la réflexion, 
alors que chez $\mathrm{V}$, ces espaces sont déjà remplis par des titres que l'on sait provisoires mais qui ne sont pas forcément considérés comme tels par les élèves, rien ne l'indiquant dans l'énoncé oral de la consigne. Dans les deux classes, la manière de réaliser le tableau de classement sert de relais à la consigne orale et contribue à fonder le contrat didactique. Mais les modalités de réalisation graphique de ces tableaux rendent la consigne plus explicite chez $\mathrm{N}$ : elle crée un espace qui invite à être rempli, la logique du tableau voulant qu'aucune case ne reste vide (Nonnon, 1991).

Pour construire le cadre de l'activité, V recourt aux deux dimensions, mémorielle et heuristique, des inscriptions au TN, tandis que $\mathrm{N}$ n'exploite que la seconde. Cette dimension heuristique fait l'objet de la partie suivante qui s'intéresse au rôle du TN pour soutenir la pensée dans l'activité de conceptualisation.

\section{Le tableau noir pour accompagner la réflexion collective}

\section{Deux modalités divergentes d'utilisation du tableau}

$\mathrm{N}$ et $\mathrm{V}$ ont distribué aux élèves une version papier du corpus d'énoncés à classer et du tableau à remplir; tous deux auront, en fin de séance, reproduit et complété cette fiche au TN. Mais le moment où chaque classe travaille sur le TN n'est pas le même. Cette variable chronogénétique a-t-elle une incidence sur l'élaboration collective du savoir?

Dans la classe de N, le TN sert d'emblée de support à une activité de recherche collective : $\mathrm{M}$ : «comment ça se fait que ça (montrant "les sapotilles sucrées") c'est dans une colonne et ça (montrant "la prédiction de Nadia") c'est dans une colonne / on voit bien que ça (montrant "Nadia") c'est différent on a même compris ce que c'était/ça (montrant "les sapotilles sucrées") et ça (montrant "la prédiction de Nadia") c'est deux colonnes différentes». Le TN prend la forme d'un écrit de travail socialisé au sein d'une communauté scientifique scolaire. Il est un «brouillon public» et le lieu d'une «écriture intermédiaire» qui, comme en témoigne le dixième état relevé dans la classe, lui confère une fonction d'aide à la pensée (Hassan, 2008) : 


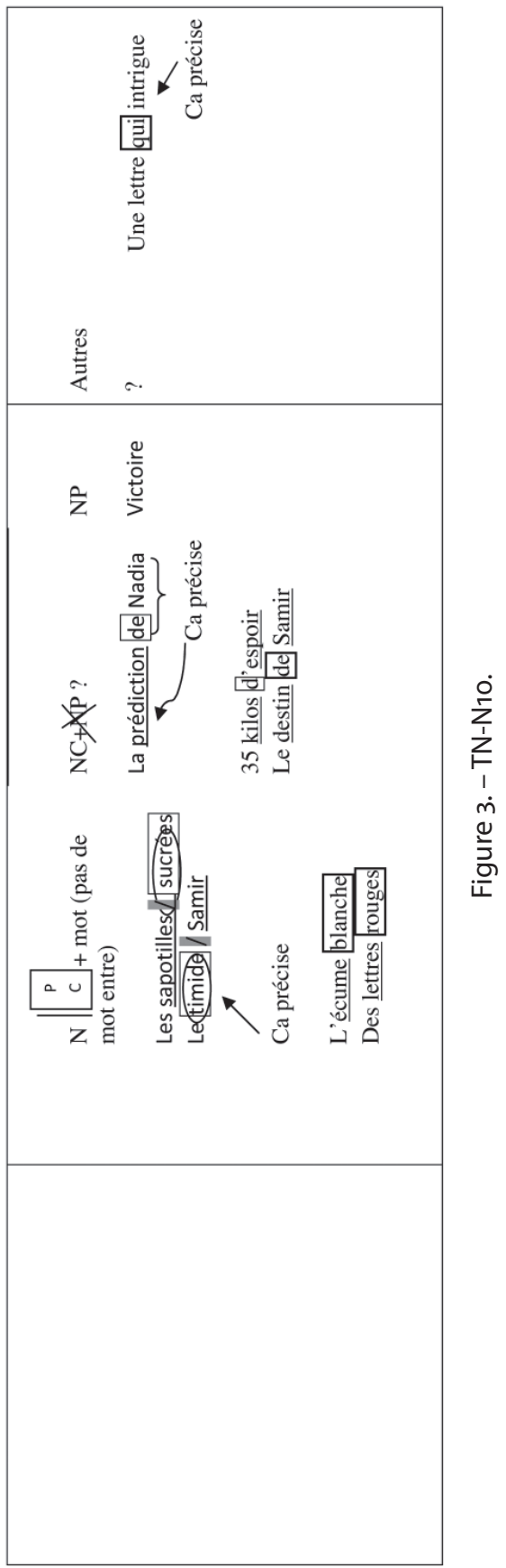


À l'issue de cette séance, $\mathrm{N}$ a reproduit l'état final du TN sur affiche. Celle-ci a servi de référence collective pour se remémorer et relancer la réflexion lors des étapes ultérieures de la séquence.

Chez V, les élèves réalisent une première fois l'activité individuellement : «tu vas faire tout seul dans ton cahier/ tu vas me donner la preuve et puis on en discutera au tableau.» Cette étape correspond chez Barth (p. 68) à «un temps de réflexion individuelle» qui permet ensuite à l'élève de participer aux échanges «à partir de son cadre de référence personnel». V met cette phase à profit pour observer les classements des élèves et ainsi préparer le guidage de la phase collective en s'appuyant sur leurs conceptions. Sa démarche renvoie à un schème d'actions proposé par Barth (p. 70) pour qui «c'est par le choix et l'ordre des exemples que l'enseignant peut influer sur les décisions de l'élève». L'activité cognitive de $\mathrm{V}$ se traduit de fait par l'ajout de deux nouveaux exemples à droite du TN central (fig. 4), pour relancer la réflexion d'élèves ayant inventé un classement qu'elle estime cohérent mais non pertinent. Mais son intervention ne repose que sur sa propre interprétation de ce classement ( «je vais en profiter pour en mettre un autre [exemple] parce que je crois que j'ai compris») et elle adopte une posture très surplombante qui court-circuite, à ce moment-là, tout échange verbal : elle infère le non-dit pour faire progresser l'activité. La phase de travail individuel débouche sur une étape au cours de laquelle la classe manipule à nouveau les énoncés du corpus, mais cette fois collectivement et avec des échanges verbaux censés placer la procédure de conceptualisation sur le mode symbolique (cf. section «Le tableau noir dans la démarche de conceptualisation»). Le TN doit servir à noter les traces des recherches individuelles pour enrichir la réflexion collective. Mais les élèves, qui ont déjà fait ce travail une fois sur papier, ne perçoivent pas cette fonction du tableau. Ils ont du mal à discuter leurs classements et à porter le regard sur d'autres propositions. D'où le reproche fait par la maitresse à l'un d'entre eux : «tu n'as eu de cesse que de faire passer ton propre classement $»$.

Le TN a été secondarisé par une affiche dans la classe $\mathrm{N}$, alors qu'il est lui-même un écrit second chez V. Ce glissement semble produire des effets très différents sur le déroulement de la démarche de conceptualisation. Avec N, la classe investit l'espace ouvert du TN qui est l'objet de nombreux retours en arrière et de reformulations se traduisant par divers types de ratures (ajouts, déplacements, suppressions, remplacements) et commentaires. Plusieurs états provisoires du tableau jalonnent les étapes de la recherche d'un critère de classement des énoncés 


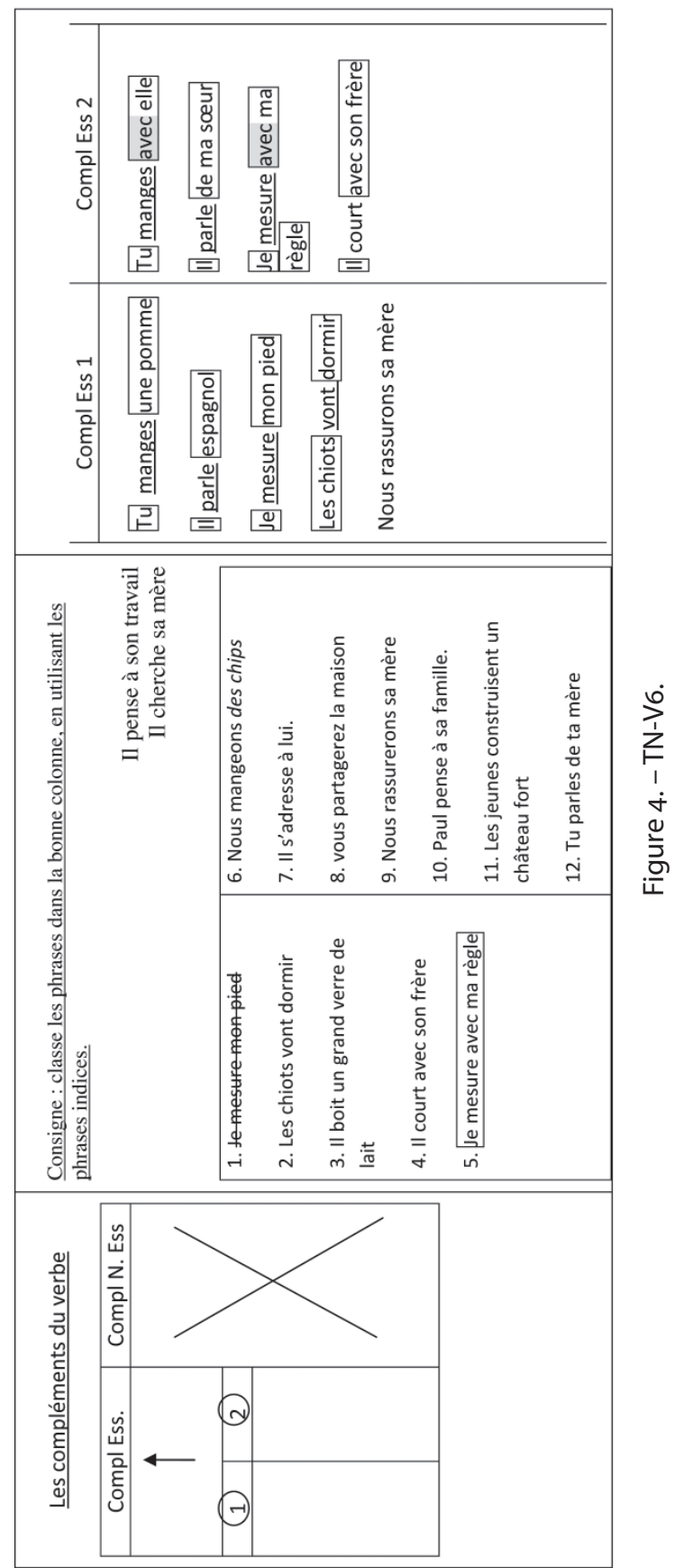


du corpus. À cette «effervescence» s'oppose un écrit plus conventionnel (cf. fig. 4) que V a sans doute davantage anticipé et qu'il associe à une routine pédagogique classique (recherche individuelle - mise en commun - institutionnalisation du savoir) davantage tournée vers la recherche de la solution attendue. C'est ici le travail individuel des élèves qui sert de brouillon à un écrit collectif - mais peu coopératif - quasi définitif, tandis que $\mathrm{N}$ fait du TN un brouillon qu'il recopiera ensuite «au propre». L'utilisation du TN par V obéit à un schème d'action assez normé, mais en définitive peu compatible avec la mise en œuvre de la démarche de conceptualisation. L'usage qu'en fait $\mathrm{N}$, sans doute plus improvisé et moins typique, s'appuie quant à lui sur des procédures de gestion du brouillon qui correspondent davantage aux caractéristiques de la démarche de conceptualisation.

\section{Le tableau chez $N$ : un support pour faire réfléchir les élèves ensemble}

On cherche à comprendre dans ce dernier paragraphe comment $\mathrm{N}$ corrèle le travail du brouillon avec la gestion des interactions. L'analyse des échanges montre en effet de fréquents phénomènes de coénonciation, parfois de sur-énonciation (Rabatel, 2004) chez les élèves, dont on peut se demander s'ils sont liés aux modalités d'utilisation du TN. Dans une étude antérieure (Sautot et Lepoire-Duc, 2009), nous avons montré que la classe $\mathrm{N}$ obéit aux règles d'une "communauté discursive scientifique scolaire» (Bernié, 2002) sémiotisée par des pratiques langagières en relation avec celles de la communauté de référence : confrontation de théories avec celles de pairs et production d'écrits de travail servant à élaborer une analyse. On situe le TN à la charnière entre ces deux dimensions de l'activité : en tant que «signal indicateur du contexte» (Nonnon, 1991), il sert de point d'appui et de référence à la communauté dont il guide la réflexion collective qui se réalise dans les échanges langagiers. De récentes théories du contexte (van Dijk, relayé par Micheli, 2006) l'abordent comme un processus objectivable pris en charge par les participants au cours même de l'interaction. Dans cette théorie, le contexte est une construction des interactants; il est évolutif et son évolution est un indice de leur activité cognitive. Il semble alors que les transformations successives du TN conditionnent la réflexion des élèves parce que leur réflexion conditionne à son tour l'évolution du TN. Dit autrement, c'est le fait de coopérer à la gestion conjointe du TN abordé comme un brouillon qui stimule l'activité cognitive et langagière des élèves. Le TN visualise un 
état de savoir à la fois complexe et en construction, et il sert de relais entre les destinataires directs et indirects (Kerbrat-Orecchioni, 1990) du polylogue scolaire. D'où une utilisation abondante de déictiques et de gestes de monstration qui permettent de se comprendre en désignant des objets langagiers que l'on ne sait pas nommer avec exactitude (M : «ça Elise nous dit ça va là̀; $\mathrm{M}$ : «on reviendra sur ce petit mot que pour l'instant vous appelez déterminant») ou de procéder, dans un premier temps, à des classements analogiques intuitifs (M: «rue des vautours il va où rue des vautours»; $\mathrm{E}:$ «dans la même [colonne]»); d'où également des échanges initiés par des élèves lorsqu'ils poursuivent l'analyse d'un élément noté au tableau que la dérive des échanges a fait perdre de vue (E: «de et qui c'est pas la même chose»; $\mathrm{M}:$ «attends je pose une question et après c'est promis je te donne la parole/ ben vas-y»). À la fin de la séance, les initiatives des élèves portent aussi bien sur des propositions spontanées de classements (E : «l'affaire Caius ça va là-bas») que sur l'énoncé des attributs d'un concept qu'ils proposent de faire figurer en en-tête du tableau (E : «entre parenthèses on met adjectif»).

L'enseignant orchestre l'activité de la classe en faisant graviter la réflexion et les interventions des élèves - qui sont impliqués, mais ne réfléchissent pas sur les mêmes objets ni au même rythme - autour du $\mathrm{TN}$ qu'il gère comme un «brouillon collectif».

\section{«Routines pédagogiques » et «routines didactiques» d'utilisation du tableau}

$\mathrm{N}$ et $\mathrm{V}$ recourent à deux modalités différentes d'utilisation du TN. On peut parler de «routine pédagogique» pour $\mathrm{V}$ qui l'utilise de manière relativement indépendante de la démarche d'apprentissage envisagée. Dans ce type de routine, les inscriptions au TN sont programmées - et dans certains cas réalisées - avant la mise en œuvre de la séance. Le $\mathrm{TN}$ est monogéré par l'enseignant et peu évolutif. Il sert donc avant tout à guider l'élève dans un programme d'activités, individuelles ou collectives, qui peut dépasser le cadre de la séance d'apprentissage. $\mathrm{N}$ s'en sert plutôt pour enrôler les élèves dans une réflexion collective. Cet enseignant n'a pas programmé les inscriptions au $\mathrm{TN}$; les premières sont minimalistes et le $\mathrm{TN}$, en tant que brouillon collectif, constitue un point de repère écrit évolutif et co-géré par le groupe classe (enseignant-élèves). On peut alors parler de «routine didactique» en ce sens 
que l'utilisation du TN est ajustée aux caractéristiques de la démarche d'apprentissage. La comparaison des deux classes montre que les «routines pédagogiques» d'utilisation du TN peuvent avoir pour effet de transformer la nature de la situation d'apprentissage; la gestion du TN semble en effet, dans le cas que nous avons étudié, faire obstacle à l'activité cognitive et communicationnelle de la classe normalement attendue lors de la mise en œuvre d'une démarche de conceptualisation. Ce constat invite à affiner les études sur le TN sous l'angle des didactiques, en vue de circonscrire et de corréler des gestes d'utilisation du TN avec des démarches d'apprentissage prototypiques. Routines «pédagogiques» et «didactiques» n'en constituent pas moins deux gestes professionnels d'égale importance et c'est au bout du compte la complémentarité entre ces deux modalités d'utilisation du TN qu'il semble nécessaire de rechercher.

\section{RÉFÉRENCES BIBLIOGRAPHIQUES}

Altet M. (2008) : «Rapport à la formation, à la pratique, aux savoirs et reconfiguration des savoirs professionnels par les stagiaires», dans $\mathrm{P}$. Perrenoud, M. Altet, C. Lessars et L. Pasquay (dir.), Conflits de savoirs en formation des enseignants, Bruxelles, De Boeck, p. 91-105.

BARTH B.-M. (1987) : L'apprentissage de l'abstraction. Méthodes pour une meilleure réussite de l'école, Paris, Retz, 255 p.

BAUTIER E. (2006) : «Le rôle des pratiques des maitres dans les difficultés scolaires des élèves : une analyse de pratiques intégrant la dimension des difficultés socialement différenciées », Recherche et Formation, $\mathrm{n}^{\circ}$ 51, Analyse de pratiques : de la recherche à la formation, $\mathrm{p} .105-118$.

BERNIÉ J.-P. (2002) : «L'approche des pratiques langagières scolaires à travers la notion de communauté discursive : un apport à la didactique comparée?», Revue française de pédagogie, $\mathrm{n}^{\circ} 141$, Vers une didactique comparée, p. 77-88.

Bonnette P. (2006) : «Le constructivisme à l'école primaire peut-il être bachelardien?», Recherches en Éducation, $\mathrm{n}^{\circ} 1$, Politiques éducatives, acteurs, institutions, p. 79-88. Disponible sur <http://recherches-eneducation.net/spip.php?article111> [consulté le 20 juillet 2010].

BOUCHARD R. (1998) : «L'interaction en classe comme polylogue praxéologique», dans F. Grossmann (éd.), 1998, Pratiques langagières et didactiques de l'écrit, Ivel-Lidilem, Grenoble 3, p. 193-210.

FillietTAz L. (2002) : La parole en action. Éléments de pragmatique psychosociale, Québec, Nota bene, 395 p. 
HASSAN R. (2008) : «L'écriture au tableau dans le travail enseignant: approche didactique», dans D. Alamargot, J. Bouchand, E. Lambert, V. Millogo et C. Beaudet (éds), Proceedings of the International Conference «de la France au Québec : l'Écriture dans tous ses états» (Poitiers, France, 12-15 novembre 2008). Disponible sur <http://www.poitoucharentes.iufm.fr/IMG/pdf/Hassan.pdf/> [consulté le 20 juillet 2010].

Kerbrat-Orecchioni C. (1990) : Les interactions verbales, tome 1, Paris, Armand Colin, 318 p.

Lepoire-Duc S. et Sautot J.-P. (2008) : «Quelques pépites d'ORL dans un

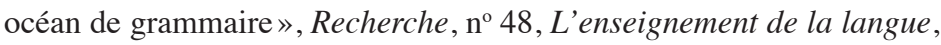
Lille, p. 77-92.

- (2009) : «La grammaire : un problème pour les élèves, un problème pour le maitre aussi », Repères, $\mathrm{n}^{\circ} 39$, La construction des savoirs grammaticaux, p. 125-142.

Mayen P. (2008) : «Intégrer les savoirs à l'action», dans P. Perrenoud, M. Altet, C. Lessars et L. Pasquay (dir.), Conflits de savoirs en formation des enseignants, Bruxelles, De Boeck, p. 44-58.

Micheli R. (2006) : «Contexte et contextualisation en analyse de discours : regard sur les travaux de T. van Dijk » [en ligne], Semen, n $^{\circ} 21$, Catégories pour l'analyse du discours politique, p. 103-120. Disponible sur $<$ http://semen .revues.org/1971> [consulté le 20 juillet 2010].

Nonnon E. (1991) : «Mettre au tableau, mettre en tableaux, ou comment structurer des discussions d'enfants : logique naturelle et formalisations écrites », Études de linguistique appliquée, $\mathrm{n}^{\circ} 81$, L'écrit dans l'oral, Didier, Paris, p. 95-117.

- (2000) : «Le tableau noir de l'enseignant, entre écrit et oral», Repères, $\mathrm{n}^{\circ} 22$, Les outils d'enseignement du français, p. 83-119.

RABATEL A. (2004) : «Déséquilibres interactionnels et cognitifs, postures énonciatives et co-construction des savoirs : co-énonciateurs, surénonciateurs et archi-énonciateurs », dans A. Rabatel (éd.), Interactions orales en contexte didactique. Mieux (se) comprendre pour mieux (se) parler et pour mieux ( $\left.s^{\prime}\right)$ apprendre, Lyon, Presses universitaires de Lyon, p. 29-66.

Sautot J.-P. et LePoire-Duc S. (2010) : Expliquer la grammaire. Comprendre les concepts de la langue, Grenoble, Scéren, coll. «Enseigner le français à l'école», 187 p.

Tава H. (1962) : Curriculum Development: Theory and Practice, New York, Harcourt, Brace and World.

VAnHulle S. (2009) : «Dire les savoirs professionnels : savoirs de référence et logiques d'action», dans R. Hofstetter et B. Schneuwly (dir.), Savoirs en (trans)formation, Bruxelles, De Boeck, p. 245-263. 